\title{
Therapeutic effects of paeonol on methyl-4-phenyl-1,2,3,6-tetrahydropyridine/probenecid-induced Parkinson's disease in mice
}

\author{
XIAOJIN SHI ${ }^{1}$, YU-HUA CHEN ${ }^{1}$, HAO LIU $^{2}$ and HONG-DANG QU ${ }^{1}$ \\ ${ }^{1}$ Department of Neurology, The First Affiliated Hospital of Bengbu Medical College, Bengbu, Anhui 233004; \\ ${ }^{2}$ Department of Pharmacy, Bengbu Medical College, Bengbu, Anhui 233030, P.R. China
}

Received August 27, 2015; Accepted May 18, 2016

DOI: $10.3892 / \mathrm{mmr} .2016 .5573$

\begin{abstract}
Paeonol is a major phenolic compound of the Chinese herb, Cortex Moutan, and is known for its antioxidant, anti-inflammatory and antitumor properties. The present study was designed to investigate the therapeutic potential and underlying mechanisms of paeonol on a 1-methyl-4-phenyl-1,2,3,6-tetrahydropyridine/probenecid (MPTP/p)-induced mouse model of Parkinson's disease (PD). MPTP (25 mg/kg), followed by probenecid $(250 \mathrm{mg} / \mathrm{kg}$ ), was administered via i.p. injection for five consecutive days to induce the mouse model of PD. Paeonol $(20 \mathrm{mg} / \mathrm{kg})$ was administrated orally for 21 days. Behavior was assessed using the rotarod performance and open-field tests. Additionally, the levels of tyrosine hydroxylase $(\mathrm{TH})$, microglia, interleukin-1 $\beta$ (IL-1 $\beta$ ), and brain-derived neurotrophic factor (BDNF) in the substantia nigra pars compacta $(\mathrm{SNpc})$ were evaluated by immunohistochemical staining. MPTP/p-induced motor deficits were observed to be significantly improved following long-term treatment with paeonol. Paeonol treatment decreased MPTP/p-induced oxidative stress, as determined by evaluating the activity levels of superoxide dismutase, catalase and glutathione. Additionally, MPTP/p-induced neuroinflammation was assessed by examining the levels of microglia and IL-1 $\beta$, which were significantly decreased following paeonol
\end{abstract}

Correspondence to: Mr. Hong-Dang Qu, Department of Neurology, The First Affiliated Hospital of Bengbu Medical College, 287 Changhuai Road, Bengbu, Anhui 233004, P.R. China

E-mail: qhd820@sohu.com

Abbreviations: BDNF, brain-derived neurotrophic factor; CAT, catalase; DA, dopamine; GSH, glutathione; Iba1, ionized calcium-binding adapter molecule 1; IL-1 $\beta$, interleukin-1 $\beta$; MPTP, methyl-4-phenyl-1,2,3,6-tetrahydropyridine; p, probenecid; PD, Parkinson's disease; SOD, superoxide dismutase; SNpc, substantia nigra pars compacta; $\mathrm{TH}$, tyrosine hydroxylase

Key words: paeonol, methyl-4-phenyl-1,2,3,6-tetrahydropyridine, Parkinson's disease, oxidative stress, neuroinflammation, tyrosine hydroxylase, brain-derived neurotrophic factor treatment. Paeonol treatment improved the MPTP/p-induced dopaminergic neurodegeneration, as measured by observing the increased TH level in the SNpc. Furthermore, the BDNF level was significantly elevated in the paeonol treatment group compared with mice treated with $\mathrm{MPTP} / \mathrm{p}$ only. In conclusion, paeonol exerted therapeutic effects in the MPTP/p-induced mouse model of PD, possibly by decreasing the damage from oxidative stress and neuroinflammation, and by enhancing the neurotrophic effect on dopaminergic neurons. The results demonstrate paeonol as a potential novel treatment for PD.

\section{Introduction}

Parkinson's disease (PD) is a progressive neurodegenerative disorder characterized by the selective degeneration of dopaminergic neurons in the substantia nigra (SN), a region of the brain that controls movement (1). The primary symptoms of $\mathrm{PD}$ include tremor at rest, muscular rigidity, bradykinesia, postural abnormalities and instability (2). Although the precise underlying pathogenic mechanism of PD remains to be elucidated, previous studies have suggested that oxidative stress, inflammation, excitotoxicity and mitochondrial dysfunction may be involved in the progression of PD $(3,4)$. Post-mortem analysis of patients with PD have indicated that oxidative stress and inflammatory pathways together cause dopaminergic neurons to undergo apoptosis, ultimately resulting in PD (5). These findings suggest that it may be beneficial to evaluate the therapeutic effects of antioxidant and anti-inflammatory agents on PD.

Although the symptoms of PD may be relieved by dopamine (DA) replacement therapy, the efficacy of drug therapy gradually declines over time (6). Furthermore, long-term treatment with L-dihydroxyphenylalanine or DA agonists results in severe adverse effects, which markedly influence patient quality of life $(7,8)$. Therapeutic strategies that unequivocally slow or stop the progression of PD do not currently exist (9). Thus, identifying an agent that provides effective protection against dopaminergic neurodegeneration would be a major breakthrough in the treatment of PD.

Agents that have neurotrophic properties may potentially promote the survival of neuronal cells and slow the progression of PD (10). Due to its neuroprotective and neurotrophic 
properties, 2'-hydroxy-4'-methoxyacetophenone (paeonol) has been identified as a potential treatment for PD. Paeonol (Fig. 1A) is a flavonoid derivative that exerts numerous physiological activities, including antioxidant (11), anti-inflammatory (12), antidiabetic (13), anticancer, analgesic, and hypnotic (14) action. An in vitro study using PC12 cells suggested that paeonol exerts anti-PD effects, as it prevented oxidative stress and the apoptosis of dopaminergic neurons (15). Another in vitro study on microglia demonstrated that paeonol inhibited the expression of inflammatory mediators, which suggests that paeonol may have therapeutic properties in neurodegenerative diseases (16). However, whether paeonol exerts therapeutic effects against 1-methyl-4-phenyl-1,2,3,6tetrahydropyridine/probenecid (MPTP/p)-induced PD in mice remains unknown.

MPTP (Fig. 1B) is a neurotoxin that induces behavioral, biochemical and neuropathological changes that are similar to those observed in patients with idiopathic PD, and it is therefore used to mimic the pathological process of PD in mice $(17,18)$. Furthermore, probenecid blocks the excretion of MPTP, which eventually results in serious neurotoxicity (19). The aim of the present study was to assess the therapeutic and neurotrophic effects of paeonol in an MTPT/p-induced mouse model of PD. Previous studies on the neuroprotective effects of paeonol revealed that doses of 20,50 and $100 \mathrm{mg} / \mathrm{kg}$ body weight significantly improved neuronal damage in experimental mice $(20,21)$; therefore, paeonol was administered at a dose of $20 \mathrm{mg} / \mathrm{kg}$ in the present study. The effects of paeonol were determined as follows: i) Evaluating the behavior of mice with rotarod performance and open-field tests; ii) measuring oxidative stress levels, specifically the activity levels of superoxide dismutase (SOD), catalase (CAT) and glutathione (GSH) in the midbrain; iii) evaluating dopaminergic neurodegeneration in the SN pars compacta (SNpc) by calculating the number of tyrosine hydroxylase (TH)-positive cells using immunohistochemical staining; iv) examining inflammatory mediators, including microglial cells and interleukin-1 $\beta$ (IL-1 $\beta$ ) using immunohistochemical staining; and v) identifying any neurotrophic properties by measuring the level of brain-derived neurotrophic factor (BDNF).

\section{Materials and methods}

Experimental animals. Adult male C57BL/6 mice $(n=40$; weight, 18-20 g) were purchased from the Comparative Medicine Center at Yangzhou University (Yangzhou, China). The animals were maintained on a 12-h light/dark cycle at $25 \pm 2{ }^{\circ} \mathrm{C}$ and $60-70 \%$ relative humidity with food and water available ad libitum in the Laboratory Animal Center of Bengbu Medical College (Bengbu, China). The study was reviewed and approved by the institutional Animal Ethical Committee of Bengbu Medical College (Bengbu, China). All surgical procedures were performed under anesthesia.

Chemicals and reagents. Paeonol (purity, $\geq 98.0 \%$ ) and MPTP hydrochloride were purchased from Sigma-Aldrich (St. Louis, MO, USA). Probenecid was obtained from Shanghai Jingchun Reagent Co., Ltd. (Shanghai, China). Rabbit anti-TH (cat. no. ab75875; 1:600), rabbit anti-IL-1 3 (cat.no. ab9722; 1:500), rabbit
anti-BDNF (cat. no. ab203573; 1:200) and rabbit anti-ionized calcium-binding adapter molecule 1 (Iba1; cat. no. ab15690; 1:200) were purchased from Abcam (Qtar, Kingdom of Saudi Arabia), and goat-anti-rabbit secondary antibody (cat. no. K5007; 1:1) was purchased from Dako (Glostrup, Denmark).

Experimental procedure. The mice were randomly divided into four groups ( $\mathrm{n}=10$ per group): i) Control group, mice were treated with saline; ii) MPTP/p only group, mice received MPTP via i.p. injection $(25 \mathrm{mg} / \mathrm{kg}$ in saline) followed by probenecid ( $250 \mathrm{mg} / \mathrm{kg}$ in $0.03 \mathrm{ml}$ dimethylsulfoxide) once a day for five consecutive days (days 1-5) to induce the PD mouse model (22); iii) $\mathrm{MPTP} / \mathrm{p}+$ paeonol group, mice received paeonol $(20 \mathrm{mg} / \mathrm{kg}$ in 5\% carboxymethylcellulose sodium) by oral gavage daily for 21 days (days 1-21) and were injected with MPTP/p (as for the $\mathrm{MPTP} / \mathrm{p}$ only group); and iv) paeonol only group, mice received oral paeonol (as for the MPTP/p+ paeonol group) for 21 days. Probenecid was injected $30 \mathrm{~min}$ prior to MPTP injection, and paeonol was administrated orally $1 \mathrm{~h}$ prior to MPTP injection. The rotarod performance and open-field tests were conducted during the course of MPTP/p administration (days 4 and 5) and during the final stages of the study (days 20 and 21). Mice were sacrificed by anesthetic overdose following the behavioral tests, and the brains were harvested for immunohistochemical and biochemical analyses (Fig. 1C).

Rotarod performance test. The rotarod performance test is widely used to assess motor and coordination abilities, particularly bradykinesia, in mice (23). In the present study, a rotarod (diameter, $3 \mathrm{~cm}$ ) at a fixed speed of $30 \mathrm{rpm}$ was utilized. The duration that each mouse spent on the revolving rod was measured. The rotating rod automatically stopped at $60 \mathrm{sec}$. If the mouse remained on the rod for the duration of the experiment, the value was recorded as $60 \mathrm{sec}$. Each mouse was trained in a separate lane of the rotarod three times a day for two consecutive days prior to performing the experiment. The rotarod test was performed on days 4 and 20, and the assessment was repeated three times for each mouse, with 30-min rest periods between tests. The mean duration for each mouse was determined and used for comparison (24).

Open-field test. The open-field test is an efficient technique for investigating the overall manifestation of motor deficits in animal models of PD, as it is sensitive to dopaminergic neuronal injury (25). In the present study, an open-field composed of a square arena $(40 \times 40 \mathrm{~cm})$ and a wall $(35-\mathrm{cm}$ high) was divided into 16 sub-squares $(4 \times 4)$. The mouse was placed in the center of the arena and the behavior of the mouse was observed for $5 \mathrm{~min}$; each mouse repeated the assessment three times. Following each test, the mice were allowed to rest for $30 \mathrm{~min}$ and the open-field was cleaned completely with a $70 \%$ ethanol solution. The number of crossings (a crossing was recorded as each time the mouse crossed the boundary of a sub-square with at least their two forepaws), grooming behaviors (rubbing the body with the paws or mouth and/or rubbing the head with the paws), rearing behaviors (standing on the hind legs), and the duration of immobility, were determined. The open-field test was performed on days 5 and 21, and conducted by an examiner who was blinded to the treatment groups. 
Assessment of SOD, CAT and GSH activity. The supernatant of the midbrain tissue homogenate was prepared by centrifugation (Smart R17; Hanil Science Industrial Co., Ltd., Incheon, South Korea) at 9,184 x g for $10 \mathrm{~min}$ at $4^{\circ} \mathrm{C}$. Total SOD activity was measured by spectrophotometry using a Total Superoxide Dismutase (T-SOD) assay kit (Nanjing Jiancheng Bioengineering Research Institute, Nanjing, China) according to the manufacturer's instructions, which contained the experimental steps and calculation formula. SOD activity is expressed as SOD U/mg protein, with one SOD unit being the quantity that reduced the absorbance at $560 \mathrm{~nm}$ by $50 \%$, according to a Synergy HT microplate reader (BioTek Instruments, Inc., Winooski, VT, USA).

CAT activity in the midbrain was measured by spectrophotometry using a CAT assay kit (Nanjing Jiancheng Bioengineering Research Institute) according to the manufacturer's instructions. The absorbance was measured at $405 \mathrm{~nm}$ on a microplate reader, and CAT activity was calculated using the formula provided by the manufacturer, and expressed as units of CAT activity/mg protein.

GSH levels were assessed by spectrophotometry using a reduced GSH assay kit (Nanjing Jiancheng Bioengineering Research Institute) according to the manufacturer's instructions. The absorbance was measured at $420 \mathrm{~nm}$ and used to calculate the GSH concentration with the formula provided by the manufacturer, and the results are expressed as GSH/mg protein.

Immunohistochemical staining procedure. Mice were anesthetized with $10 \%$ chloral hydrate $(3 \mathrm{ml} / \mathrm{kg}$; Sigma-Aldrich) following the behavioral test and their hearts were perfused with saline, followed by $4 \%$ paraformaldehyde for $20 \mathrm{~min}$. The brains were embedded in paraffin and coronally sectioned through the SNpc region at a thickness of $4 \mu \mathrm{m}$. Sections were fixed on slides, deparaffinized with three changes of xylene, and then dehydrated through decreasing grades of absolute alcohol (95, 75 and 50\%). Endogenous peroxidases were blocked by incubating the sections with $3 \% \mathrm{H}_{2} \mathrm{O}_{2}$ for $10 \mathrm{~min}$. Following antigen retrieval with citrate salt buffer ( $\mathrm{pH} 7.0$; Beijing Zhongshan Golden Bridge Biotechnology Co., Ltd., Beijing, China) in microwave oven for $6 \mathrm{~min}$ and rinsing with phosphate-buffered saline, the sections were incubated at $37^{\circ} \mathrm{C}$ overnight with a primary monoclonal antibody (rabbit anti-TH, rabbit anti-IL-1 $\beta$, rabbit anti-Iba1 or rabbit anti-BDNF), and then with a biotinylated goat anti-rabbit secondary antibody for $50 \mathrm{~min}$. Sections were subsequently washed and developed with a 3,3'-diaminobenzidine staining kit (Dako); the chromogenic reaction was terminated when brown granules (positive cells) were observed. Finally, the sections were counterstained with hematoxylin, rinsed with deionized water, dried and sealed with neutral balsam. The stained sections were analyzed under an optical microscope (Nikon Corporation, Tokyo, Japan).

Statistical analysis. All data are expressed as the mean \pm standard deviation. Multiple comparisons analyses were performed using a one-way analysis of variance followed by Tukey's post-hoc tests using the Statistical Package for the Social Sciences (SPSS) software version 16.0 (SPSS, Inc., Chicago, IL, USA). $\mathrm{P}<0.05$ was considered to indicate a statistically significant difference.
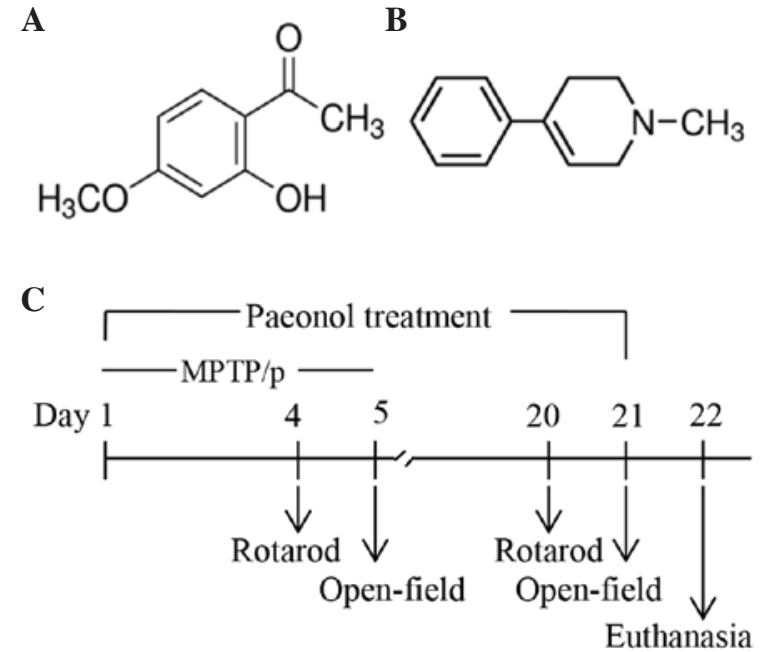

Figure 1. Structures of (A) paeonol and (B) MPTP, and (C) a schematic of the experimental procedure. MPTP, methyl-4-phenyl-1,2,3,6-tetrahydropyridine; p, probenecid.

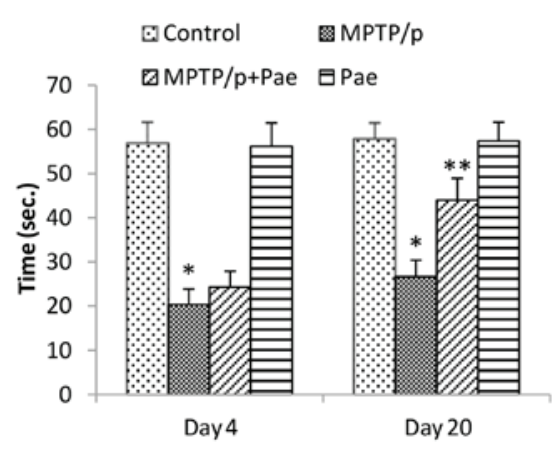

Figure 2. Outcomes of the rotarod performance test (retention time) by control and experimental mice. The MPTP/p group showed significantly reduced retention times compared with the control group on days 4 and 20. The $\mathrm{MPTP} / \mathrm{p}+$ Pae group showed more staining on day 20 when compared with the MPTP/p only group, although this difference was not observed on day 4 . The retention times of the Pae only group were similar to those of the control group. Data are expressed as the mean \pm standard deviation, and statistical analysis was performed using a one-way analysis of variance followed by Tukey's post-hoc test. ${ }^{*} \mathrm{P}<0.01$ vs. control; ${ }^{* *} \mathrm{P}<0.01$ vs. MPTP/p only. MPTP, methyl-4-phenyl-1,2,3,6-tetrahydropyridine; p, probenecid; Pae, paeonol.

\section{Results}

Effect of paeonol on the behavior of mice with $M P T P / p$-induced $P D$ in the rotarod performance test. The MPTP/p group demonstrated significantly reduced retention times compared with the control group on days 4 and $20(\mathrm{P}<0.01)$. The MPTP/p +paeonol group demonstrated significant improvements in motor performance on day 20 compared with the MPTP/p only group $(\mathrm{P}<0.01)$, although no significant improvement was observed on day 4 . The behavioral performances of the paeonol only group were similar to those of the control group (Fig. 2).

Effect of paeonol on the behavior of mice with MPTP/p-induced $P D$ in the open-field test. The results of the open-field test are presented in Fig. 3. The MPTP/p only group exhibited significantly reduced locomotor activity (as measured by lines 
A
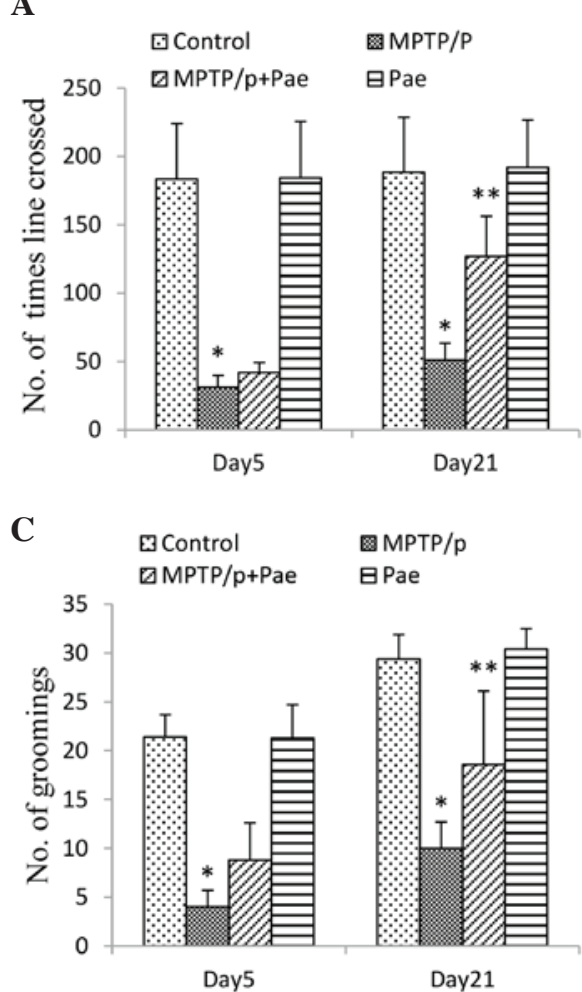

B

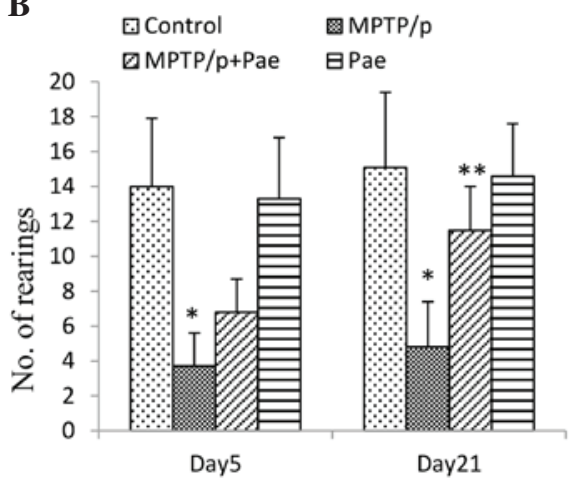

D

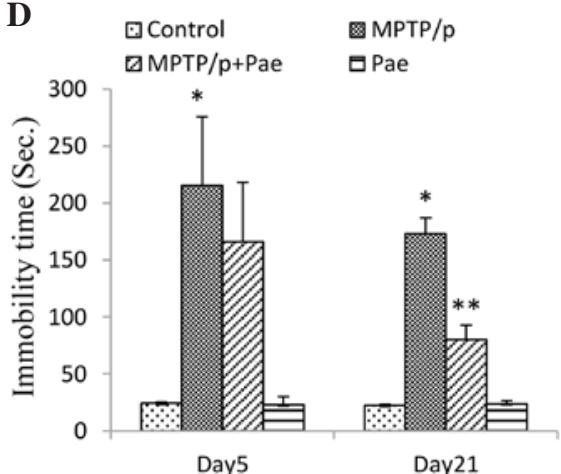

Figure 3. Performances in the open-field test by control and experimental mice. Parameters measured included (A) line crossings, (B) rearings, (C) grooming activities, and (D) immobility time. All types of movement were significantly reduced, and immobility time was significantly increased by MPTP/p treatment when compared with the control group on days 5 and 21 ; however, this reduction in motor activity was attenuated by treatment with Pae on day 21 . No significant differences were observed between the control and the Pae only groups. Data are expressed as the mean \pm standard deviation, and statistical analysis was performed using a one-way analysis of variance. $\mathrm{P}<0.01$ vs. control; ${ }^{* *} \mathrm{P}<0.01 \mathrm{vs}$. MPTP/p only. MPTP, methyl-4-phenyl-1,2,3,6-tetrahydropyridine; p, probenecid; Pae, paeonol.
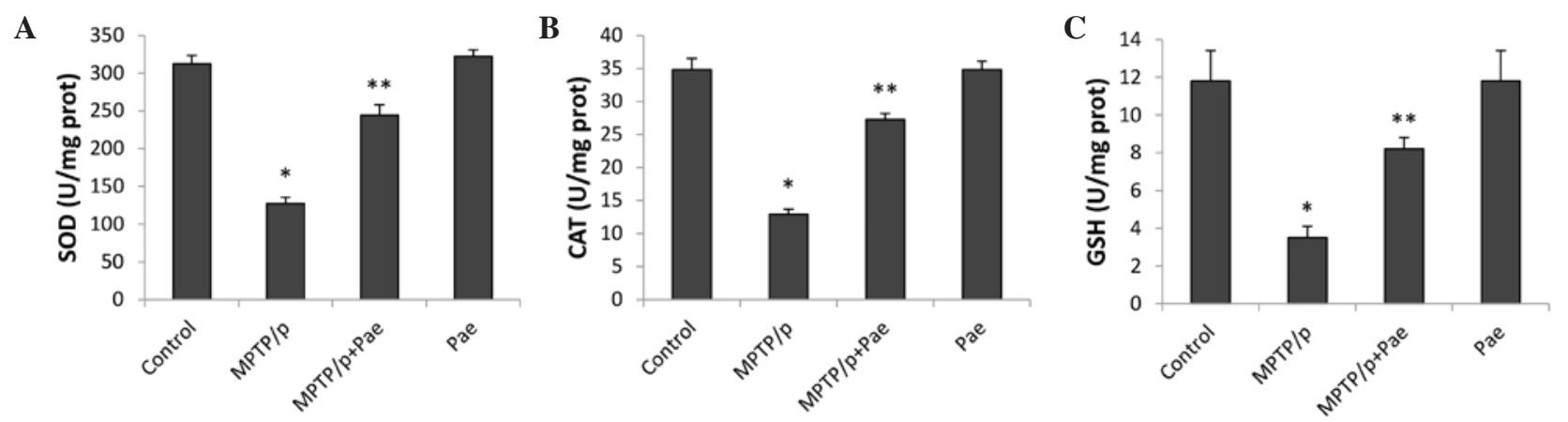

Figure 4. Effects of Pae on the activity levels of SOD, CAT, and GSH in the midbrain of control and experimental mice. The levels of (A) SOD, (B) CAT and (C) GSH were significantly reduced in the MPTP/p only group compared with the control group. Treatment with Pae significantly restored the activity of SOD, CAT and GSH in the MPTP/p + Pae group compared with the MPTP/p only group $(\mathrm{P}<0.01)$. No significant differences were observed between the control and the Pae only groups. Data are expressed as the mean \pm standard deviation, and statistical analysis was performed using a one-way analysis of variance. "P<0.01 vs. control; ${ }^{* *} \mathrm{P}<0.01$ vs. MPTP/p only. SOD, superoxide dismutase; CAT, catalase; GSH, glutathione; MPTP, methyl-4-phenyl-1,2,3,6-tetrahydropyridine; p, probenecid; Pae, paeonol.

crossed; Fig. 3A), rearing (Fig. 3B) and grooming (Fig. 3C), and increased duration of immobility (Fig. 3D), compared with the control group on days 5 and $21(\mathrm{P}<0.01)$. The MPTP/p + paeonol group demonstrated significantly improved locomotor activity, rearing and grooming, and decreased immobility time compared with the MPTP/p only group on day $21(\mathrm{P}<0.01)$; however, no significant differences were observed on day 5 . Furthermore, no significant differences were observed between the control and the paeonol only groups.
Paeonol attenuated MPTP/p-induced oxidative stress. The activity levels of SOD, CAT and GSH in the midbrain, which reflect the level of oxidative stress, are presented in Fig. 4. MPTP/p significantly reduced the levels of SOD, CAT and $\mathrm{GSH}$, and therefore enhanced the level of oxidative stress in the MPTP/p only group compared with the control group $(\mathrm{P}<0.01)$. Treatment with paeonol for 21 days significantly restored the activity of SOD, CAT and GSH, and therefore alleviated oxidative stress in the MPTP/p + paeonol group 
A

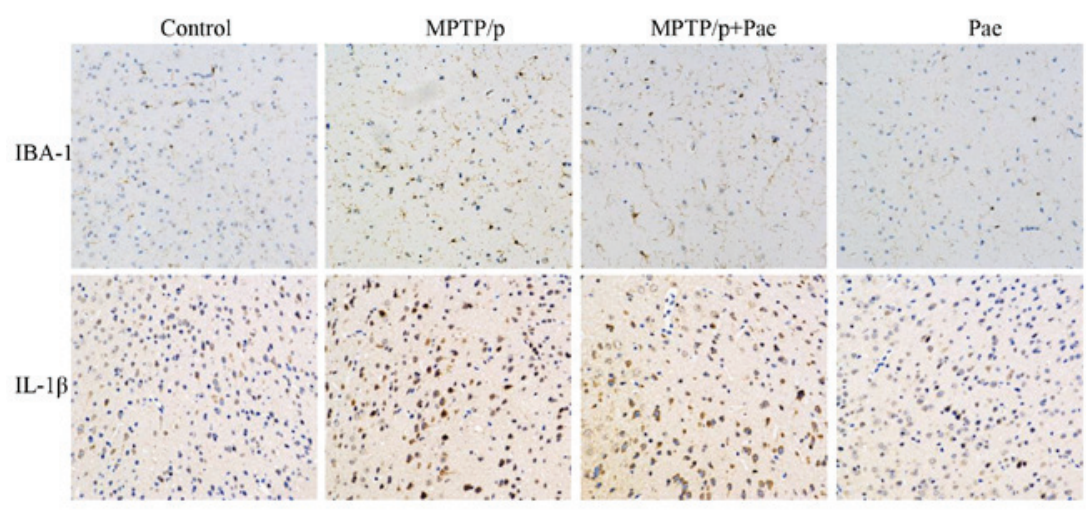

B

\section{Iba1}

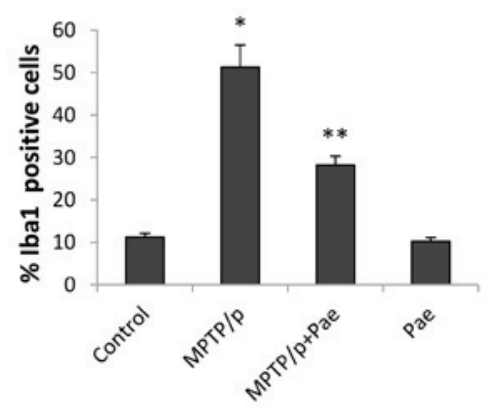

IL-1 $\beta$

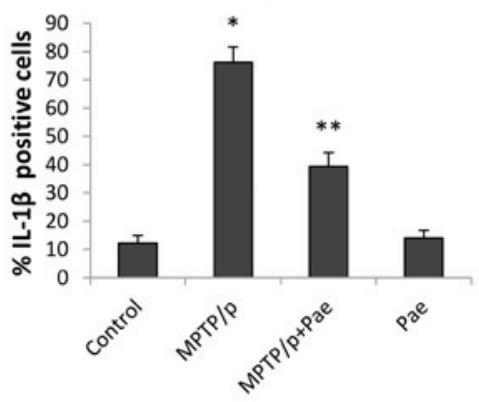

Figure 5. Effects of paeonol on Iba1- and IL-1 $\beta$-positive cells in the SNpc of control and experimental mice. (A) Immunohistochemical staining of Iba1 and IL-1 $\beta$ in the SNpc (magnification, $x 200$ ) reveals that the administration of MPTP/p increased the expression levels of Iba1 and IL-1 $\beta$. This effect was alleviated by treatment with paeonol. (B) Quantification of Iba1 and IL-1 $\beta$ staining was performed by counting the number of Iba1- and IL-1 $\beta$-positive cells in the SNpc. The mean value was expressed as the ratio of relative positive cells to the total number of cells. Data are expressed as the mean \pm standard deviation. ${ }^{*}<0.01$ vs. control; ${ }^{* *} \mathrm{P}<0.01$ vs. MPTP/p only. Iba1, ionized calcium-binding adapter molecule $1 ;$ IL-1 $\beta$, interleukin-1 $\beta$; SNpc, substantia nigra pars compacta; MPTP, methyl-4-phenyl-1,2,3,6-tetrahydropyridine; p, probenecid; Pae, paeonol.

compared with the MPTP/p only group $(\mathrm{P}<0.01)$. No significant differences were observed between the control and the paeonol only groups.

Paeonol attenuated MPTP/p-induced neuroinflammation. Microglia were stained with an antibody against Iba1 (a microglial marker). As presented in Fig. 5A, immunohistochemical staining for Ibal and IL-1 $\beta$ in the SNpc revealed large brown-colored regions in the MPTP/p only group. However, the positive cells in the MPTP/p + paeonol group were not only smaller, but were also lighter in color than those in the MPTP/p only group. No or little specific staining of Iba1 or IL-1 $\beta$ was observed in the control and the paeonol only groups. The numbers of Iba1-positive (51 vs. 11\%; $\mathrm{P}<0.01)$ and IL-1 $\beta$-positive (76 vs. $12 \%$; $\mathrm{P}<0.01$ ) cells significantly increased in the $\mathrm{MPTP} / \mathrm{p}$ only group compared with the control group. However, the numbers of Iba1-positive ( 28 vs. $51 \%$; $\mathrm{P}<0.01$ ) and IL-1 $\beta$-positive (39 vs. $76 \% ; \mathrm{P}<0.01)$ cells significantly decreased in the MPTP/p + paeonol group compared with the $\mathrm{MPTP} / \mathrm{p}$ only group. No significant differences were observed between the paeonol only and control groups.

Paeonol attenuated the MPTP/P-induced loss of dopaminergic neurons. The results of the immunohistochemical examinations revealed that the majority of the TH-positive cells (representing dopaminergic neurons) in the SNpc were fusiform or had an irregular triangular shape, and dense neurons with dark-stained cytoplasms were observed in the control and paeonol only groups (Fig. 6A). MPTP administration induced clear fragmentation of the dopaminergic neurons, causing the cellular outline to be ambiguous and the cytoplasmic staining to be faint (Fig. 6B); thus, only $19 \%$ of the TH-positive cells were observed in the $\mathrm{SN}$ of the MPTP/p only group when compared with $100 \%$ of the TH-positive cells in the control group $(\mathrm{P}<0.01$; Fig. 6B). However, the MPTP/p + paeonol group demonstrated protection of TH-positive cells when compared with the MPTP/p only group (51 vs. 19\%; $\mathrm{P}<0.01$ ). No significant difference was observed between the paeonol only and the control groups.

Paeonol attenuated the MPTP/p-induced loss of BDNF. $\mathrm{MPTP} / \mathrm{p}$ administration significantly reduced the level of $\mathrm{BDNF}$ in the SN of the MPTP/p only group compared with the level in the control group (19 vs. 49\%; $\mathrm{P}<0.01$; Fig. 7). Treatment with paeonol for 21 days significantly restored the level of BDNF in the SN of mice with MPTP/p-induced PD compared with the level in the MPTP/p only group (36 vs. $19 \%$; $\mathrm{P}<0.01$ ). The level of BDNF in the paeonol only group was slightly increased compared with the level in the control group; however, the difference was not statistically significant.

\section{Discussion}

The discovery of neuroprotective compounds that slow or stop the progression of neurodegeneration is essential for treating various neurodegenerative diseases, including PD $(26,27)$. The results of the present study demonstrate for the first time, to the best of our knowledge, that long-term paeonol treatment 
A

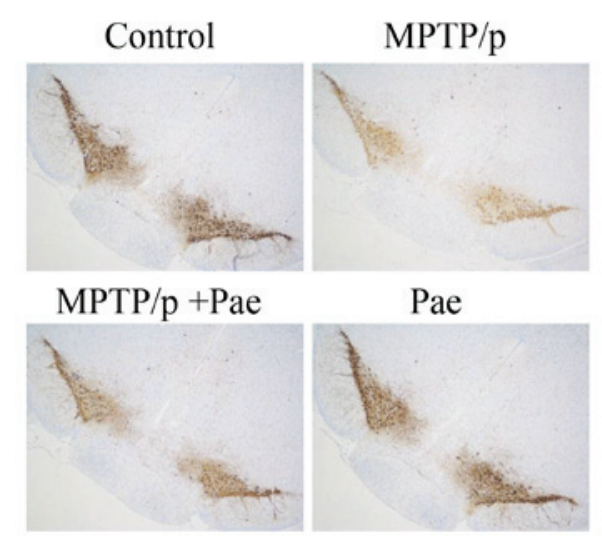

B

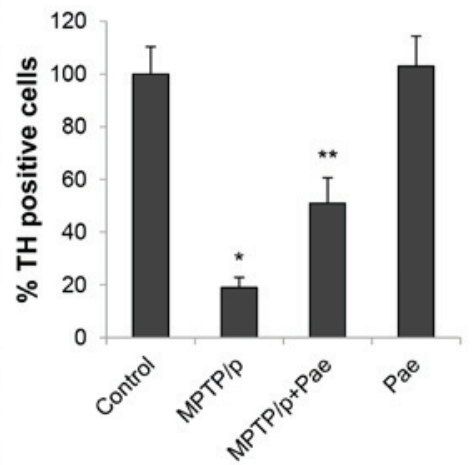

Figure 6. Effects of paeonol on the expression level of $\mathrm{TH}$ in the SNpc of control and experimental mice. (A) Immunohistochemical staining of TH (magnification, $\mathrm{x} 40$ ) reveals that the administration of MPTP/p reduced the expression of TH, while co-administration of paeonol and MPTP/p attenuated the loss of TH. (B) Quantification of TH staining was performed by counting the number of TH-positive cells in the SNpc. The mean value for TH-positive cells was expressed as a percentage of that in the matched control mice. Data are expressed as the mean \pm standard deviation. ${ }^{*} \mathrm{P}<0.01$ vs. control; ${ }^{* *} \mathrm{P}<0.01$ vs. MPTP/p only. TH, tyrosine hydroxylase; SNpc, substantia nigra pars compacta; MPTP, methyl-4-phenyl-1,2,3,6-tetrahydropyridine; p, probenecid; Pae, paeonol.

A

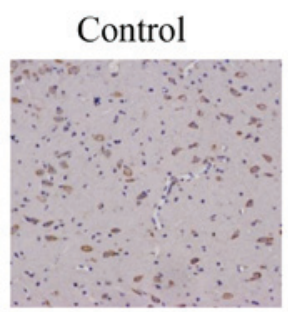

$\mathrm{MPTP} / \mathrm{p}+\mathrm{Pae}$

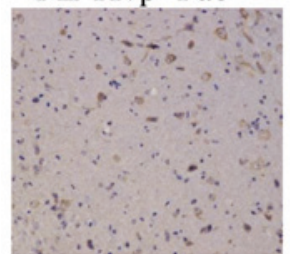

$\mathrm{MPTP} / \mathrm{p}$

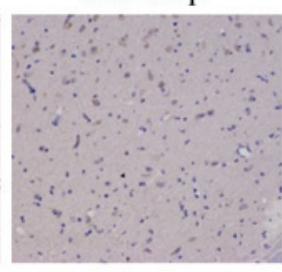

$\mathrm{Pae}$

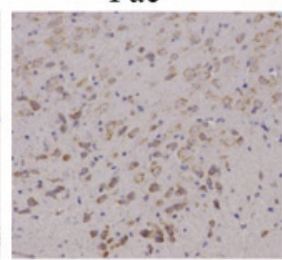

B

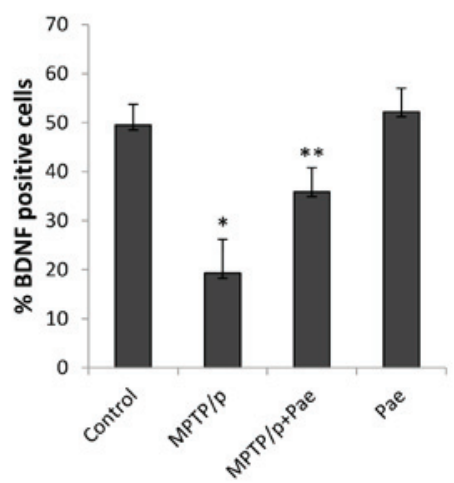

Figure 7. Effects of paeonol on BDNF-positive cells in the SNpc of control and experimental mice. (A) Immunohistochemical staining of BDNF (magnification, $\mathrm{x} 200$ ) in the SNpc reveals that the administration of MPTP/p reduced the BDNF expression, and co-administration of paeonol and MPTP/p attenuated the loss of BDNF. (B) Quantification of BDNF staining was performed by counting the number of BDNF-positive cells in the SNpc. The mean value for BDNF-positive cells was expressed as the ratio of relative positive cells to the number of total cells. Data are expressed as the mean \pm standard deviation. "P<0.01 vs. control; ${ }^{* *} \mathrm{P}<0.01$ vs. MPTP/p only. BDNF, brain-derived neurotrophic factor; SNpc, substantia nigra pars compacta; MPTP, methyl-4-phenyl-1,2,3,6-tetrahydropyridine; p, probenecid; Pae, paeonol.

exhibits therapeutic effects in mice with MPTP/p-induced PD. Mice experienced improvements in motor dysfunction, suppression of oxidative stress and the inflammatory cascade, and protection of TH-positive neurons. In addition, it was observed that BDNF levels increased following paeonol treatment, suggesting that paeonol exerts neurotrophic effects on PD.

In the present study, the mouse model of PD was induced by co-administration of MPTP and probenecid, which was more suitable for investigating the therapeutic effects of long-term drug treatment than mouse models of PD induced with MPTP alone (28). MPTP effectively crosses the blood-brain barrier and accumulated MPTP is metabolized into 1-methyl-4-phenylpyridinium (MPP+) by monoamine oxidase-B. MPP+, which inhibits complex I of the mitochondrial respiratory chain, enters dopaminergic neurons via the dopamine transporter, ultimately inducing their degeneration $(29,30)$.

There is increasing evidence that the motor dysfunctions of MPTP/p-treated mice may be observed using behavioral tests, including the rotarod performance and open-field tests (31). In the present study, the performances of mice with PD significantly improved on the rotarod performance and open-field tests following long-term treatment with paeonol, although no apparent improvement was observed on day 4. This is consistent with the results of previous studies $(28,32)$. The results of the behavioral tests suggested that long-term paeonol treatment has a therapeutic effect on mice with MPTP/p-induced PD.

Oxidative stress is widely considered to be important in the development of PD $(33,34)$. Extensive post-mortem studies have indicated that oxidative stress induces the downregulation of antioxidant protective systems, including 
SOD, GSH and CAT, and damages lipids, proteins and DNA, ultimately resulting in damage to dopaminergic neurons in the SNpc (34-36). SOD, CAT and GSH effectively remove oxygen free radicals and lipid peroxides, which may be used to measure the level of oxidative stress (37). In the present study, the activity levels of SOD, CAT and GSH were significantly decreased in the MPTP/p only group, suggesting that oxidative stress is involved in the pathogenesis of PD. Following long-term paeonol treatment, the levels of SOD, CAT, and GSH significantly improved in mice with MPTP/p-induced PD, which is consistent with the findings of a previous study (38). The results of the present study suggest that paeonol exerts a therapeutic effect on MPTP/p-induced oxidative stress, perhaps due to its antioxidant activity and ability to reduce the toxicity caused by oxidative stress.

Excessive microglial activation has been observed in the SN of human post-mortem tissue from PD patients and animal models, suggesting that neuroinflammation and activated microglia are crucial in the pathogenesis of PD (39-41). Microglia are involved in immune defense and surveillance, and activate a variety of proinflammatory cytokines, including IL-1 $\beta$, which may directly induce apoptosis in dopaminergic neurons $(42,43)$. Studies have demonstrated that the overactivation of microglia and the overproduction of proinflammatory cytokines may enhance oxidative stress, eventually leading to neuronal death $(44,45)$. In addition, a previous study has revealed that paeonol may be a potential neuroprotective agent, possibly through inhibiting microglia-mediated inflammation and oxidative stress-induced neuronal damage (46). In the present study, the levels of microglia and IL-1 $\beta$ were significantly increased in the SNpc of mice in the MPTP/p only group. However, long-term paeonol treatment significantly decreased these levels. These results suggest that paeonol was therapeutic, perhaps by downregulating the inflammatory cascade.

$\mathrm{TH}$ is the limiting enzyme of dopamine synthesis, and TH immunohistochemical analysis measures the number and function of dopaminergic neurons and fibers in the SN, as reductions in dopaminergic neurons may be the primary reason for the onset of $\mathrm{PD}(47,48)$. In the present study, immunohistochemical analysis of $\mathrm{TH}$ revealed a significant loss of dopaminergic neurons in the SNpc of mice in the $\mathrm{MPTP} / \mathrm{p}$ only group, perhaps due to MPTP-induced oxidative stress and inflammation (49). Long-term paeonol treatment appeared to protect dopaminergic neurons and fibers in mice with MPTP/p-induced PD, which suggests that its therapeutic effects may be associated with its anti-inflammatory and antioxidant effects.

BDNF is a member of a family of neurotrophic factors that has attracted widespread attention, as it exhibits strong nutritional and protective effects, particularly in DA neurons $(50,51)$. Studies have demonstrated that the level of BDNF significantly decreases in patients with PD $(52,53)$, which is consistent with the results of the present study. These findings suggest that novel treatment strategies for PD should aim to increase the expression levels of BDNF. In the present study, it was observed that treatment with paeonol enhanced BDNF expression levels and the number of TH-positive cells in the SN of mice with MPTP/p-induced PD, while no apparent improvement was observed in the paeonol only group. These findings suggest that the therapeutic effects of paeonol may be associated with its potential neurotrophic effects on dopaminergic neurons.

In conclusion, the results of the present study demonstrate that paeonol exerts therapeutic effects against MPTP/p-induced $\mathrm{PD}$ in mice, as revealed by improvements in behavioral tests and enhanced TH expression compared with mice that did not receive paeonol. In addition, the therapeutic effects may be associated with the ability of paeonol to reduce oxidative stress, increase antioxidant defenses, and inhibit the overactivation of microglia along with the release of proinflammatory factors. Furthermore, BDNF levels were improved in mice with $\mathrm{MPTP} / \mathrm{p}$-induced PD following long-term treatment with paeonol, indicating that paeonol has neurotrophic effects on dopaminergic neurons. Therefore, the results of the present study suggest that paeonol has potential as a treatment for PD; however, the dose of paeonol $(20 \mathrm{mg} / \mathrm{kg})$ administered in the present study may not be directly extrapolated to humans. Future studies are required to investigate the dose-dependent effect on MPTP/p-induced PD mice and determine an optimal dose. In addition, further studies are necessary to establish the exact mechanisms underlying the effects of paeonol.

\section{Acknowledgements}

The present study was supported by the Fund of Education Department of Anhui (grant no. KJ2014A163) and as a Postgraduate Research and Innovation Project of Bengbu Medical College (grant no. Byycx1406).

\section{References}

1. Meissner WG, Frasier M, Gasser T, Goetz CG, Lozano A, Piccini P, Obeso JA, Rascol O, Schapira A, Voon V, et al: Priorities in Parkinson's disease research. Nat Rev Drug Discov 10: 377-393, 2011.

2. Kowal SL, Dall TM, Chakrabarti R, Storm MV and Jain A: The current and projected economic burden of Parkinson's disease in the United States. Mov Disord 28: 311-318, 2013.

3. Guillot TS, Richardson JR, Wang MZ, Li YJ, Taylor TN, Ciliax BJ, Zachrisson O, Mercer A and Miller GW: PACAP38 increases vesicular monoamine transporter 2 (VMAT2) expression and attenuates methamphetamine toxicity. Neuropeptides 42 : 423-434, 2008

4. Klegeris A and McGeer PL: R-(-)-Deprenyl inhibits monocytic THP-1 cell neurotoxicity independently of monoamine oxidase inhibition. Exp Neurol 166: 458-464, 2000.

5. Hartmann A: Postmortem studies in Parkinson's disease. Dialogues Clin Neurosci 6: 281-293, 2004.

6. Birkmayer W and HornykiewiczO: The L-3,4-dioxyphenylalanine (DOPA)-effect in Parkinson-akinesia. Wien Klin Wochenschr 73: 787-788, 1961 (In German).

7. Morin N, Morissette M, Grégoire L, Rajput A, Rajput AH and Di Paolo T: Contribution of brain serotonin subtype $1 \mathrm{~B}$ receptors in levodopa-induced motor complications. Neuropharmacology 99: 356-368, 2015.

8. Winner B, Desplats P, Hagl C, Klucken J, Aigner R, Ploetz S Laemke J, Karl A, Aigner L, Masliah E, et al: Dopamine receptor activation promotes adult neurogenesis in an acute Parkinson model. Exp Neurol 219: 543-552, 2009.

9. Abdel-Salam OM: Drugs used to treat Parkinson's disease, present status and future directions. CNS Neurol Disord Drug Targets 7: 321-342, 2008.

10. Tan W, Xue-bin C, Tian Z, Xiao-wu C, Pei-pei H, Zhi-bin C and Bei-sha T: Effects of simvastatin on the expression of inducible nitric oxide synthase and brain-derived neurotrophic factor in a lipopolysaccharide-induced rat model of Parkinson disease. Int $\mathrm{J}$ Neurosci 126: 278-286, 2016.

11. Chen B, Ning M and Yang G: Effect of paeonol on antioxidant and immune regulatory activity in hepatocellular carcinoma rats. Molecules 17: 4672-4683, 2012. 
12. Fu PK, Wu CL, Tsai TH and Hsieh CL: Anti-inflammatory and anticoagulative effects of paeonol on LPS-induced acute lung injury in rats. Evid Based Complement Alternat Med 2012: 837513, 2012.

13. Lau CH, Chan CM, Chan YW, Lau KM, Lau TW, Lam FC, Law WT, Che CT, Leung PC, Fung KP, et al: Pharmacological investigations of the anti-diabetic effect of Cortex Moutan and its active component paeonol. Phytomedicine 14: 778-784, 2007.

14. Li M, Tan SY and Wang XF: Paeonol exerts an anticancer effect on human colorectal cancer cells through inhibition of $\mathrm{PGE}_{2}$ synthesis and COX-2 expression. Oncol Rep 32: 2845-2853, 2014

15. Wang H, Geng ZM, Hu ZW, Wang SY and Zhao B: Neuroprotective effects of paeonol in a cell model of Parkinson disease. Zhejiang Da Xue Xue Bao Yi Xue Ban 44: 30-36, 2015 (In Chinese).

16. Lin C, Lin HY, Chen JH, Tseng WP, Ko PY, Liu YS, Yeh WL and Lu DY: Effects of paeonol on anti-neuroinflammatory responses in microglial cells. Int J Mol Sci 16: 8844-8860, 2015.

17. Mounsey RB, Mustafa S, Robinson L, Ross RA, Riedel G, Pertwee RG and Teismann P: Increasing levels of the endocannabinoid $2-\mathrm{AG}$ is neuroprotective in the 1-methyl-4-phenyl-1,2,3,6-tetrahydropyridine mouse model of Parkinson's disease. Exp Neurol 273: 36-44, 2015.

18. He XJ and Nakayama H: Transiently impaired neurogenesis in MPTP mouse model of Parkinson's disease. Neurotoxicology 50: 46-55, 2015.

19. Lau YS, Patki G, Das-Panja K, Le WD and Ahmad SO: Neuroprotective effects and mechanisms of exercise in a chronic mouse model of Parkinson's disease with moderate neurodegeneration. Eur J Neurosci 33: 1264-1274, 2011.

20. Hsieh CL, Cheng CY, Tsai TH, Lin IH, Liu CH, Chiang SY, Lin JG, Lao CJ and Tang NY: Paeonol reduced cerebral infarction involving the superoxide anion and microglia activation in ischemia-reperfusion injured rats. J Ethnopharmacol 106 : 208-215, 2006.

21. Zhong SZ, Ge QH, Qu R, Li Q and Ma SP: Paeonol attenuates neurotoxicity and ameliorates cognitive impairment induced by d-galactose in ICR mice. J Neurol Sci 277: 58-64, 2009.

22. Petroske E, Meredith GE, Callen S, Totterdell S and Lau YS: Mouse model of Parkinsonism: A comparison between subacute MPTP and chronic MPTP/probenecid treatment. Neuroscience 106: 589-601, 2001.

23. Tarantini S, Hertelendy P, Tucsek Z, Valcarcel-Ares MN, Smith N, Menyhart A, Farkas E, Hodges EL, Towner R, Deak F, et al: Pharmacologically-induced neurovascular uncoupling is associated with cognitive impairment in mice. J Cereb Blood Flow Metab 35: 1871-1881, 2015.

24. Carter RJ, Morton J and Dunnett SB: Motor coordination and balance in rodents. Curr Protoc Neurosci Unit 8.12, 2001.

25. Sedelis M, Hofele K, Auburger GW, Morgan S, Huston JP and Schwarting RK: MPTP susceptibility in the mouse: Behavioral, neurochemical, and histological analysis of gender and strain differences. Behav Genet 30: 171-182, 2000.

26. Aguirre-Vidal Y, Montes S, Tristan-López L, Anaya-Ramos L, Teiber J,Ríos C, Baron-Flores V and Monroy-Noyola A: The neuroprotective effect of lovastatin on MPP(+)-induced neurotoxicity is not mediated by PON2. Neurotoxicology 48: 166-170, 2015.

27. Palencia G, Garcia E, Osorio-Rico L, Trejo-Solís C, Escamilla-Ramírez A and Sotelo J: Neuroprotective effect of thalidomide on MPTP-induced toxicity. Neurotoxicology 47: 82-87, 2015.

28. Patil SP, Jain PD, Ghumatkar PJ, Tambe R and Sathaye S: Neuroprotective effect of metformin in MPTP-induced Parkinson's disease in mice. Neuroscience 277: 747-754, 2014.

29. Jackson-Lewis V, Blesa J and Przedborski S: Animal models of Parkinson's disease. Parkinsonism Relat Disord 18 (Suppl 1): S183-S185, 2012.

30. Zang LY and Misra HP: Generation of reactive oxygen species during the monoamine oxidase-catalyzed oxidation of the neurotoxicant, 1-methyl-4-phenyl-1,2,3,6-tetrahydropyridine. J Biol Chem 268: 16504-16512, 1993.

31. Patil SP, Jain PD, Sancheti JS, Ghumatkar PJ, Tambe R and Sathaye S: Neuroprotective and neurotrophic effects of Apigenin and Luteolin in MPTP induced parkinsonism in mice. Neuropharmacology 86: 192-202, 2014.

32. Wang XL, Xing GH, Hong B, Li XM, Zou Y, Zhang XJ and Dong MX: Gastrodin prevents motor deficits and oxidative stress in the MPTP mouse model of Parkinson's disease: Involvement of ERK1/2-Nrf2 signaling pathway. Life Sci 114: 77-85, 2014.
33. Gaki GS and Papavassiliou AG: Oxidative stress-induced signaling pathways implicated in the pathogenesis of Parkinson's disease. Neuromolecular Med 16: 217-230, 2014.

34. Zhang J, Perry G, Smith MA, Robertson D, Olson SJ, Graham DG and Montine TJ: Parkinson's disease is associated with oxidative damage to cytoplasmic DNA and RNA in substantia nigra neurons. Am J Pathol 154: 1423-1429, 1999.

35. Jenner P and Olanow CW: Oxidative stress and the pathogenesis of Parkinson's disease. Neurology 47 (6 Suppl 3): S161-S170, 1996.

36. Maj MC, Tkachyova I, Patel P, Addis JB, Mackay N, Levandovskiy V, Lee J, Lang AE, Cameron JM and Robinson BH: Oxidative stress alters the regulatory control of p66Shc and Akt in PINK1 deficient cells. Biochem Biophys Res Commun 399: 331-335, 2010.

37. Weydert CJ and Cullen JJ: Measurement of superoxide dismutase, catalase and glutathione peroxidase in cultured cells and tissue. Nat Protoc 5: 51-66, 2010.

38. Selvakumar GP, Janakiraman U, Essa MM, Justin Thenmozhi A and Manivasagam T: Escin attenuates behavioral impairments, oxidative stress and inflammation in a chronic MPTP/probenecid mouse model of Parkinson's disease. Brain Res 1585: 23-36, 2014.

39. Tansey MG and Goldberg MS: Neuroinflammation in Parkinson's disease: Its role in neuronal death and implications for therapeutic intervention. Neurobiol Dis 37: 510-518, 2010.

40. Hirsch EC, Hunot S, Damier P and Faucheux B: Glial cells and inflammation in Parkinson's disease: A role in neurodegeneration? Ann Neurol 44 (3 Suppl 1): S115-S120, 1998.

41. Hirsch EC and Hunot S: Neuroinflammation in Parkinson's disease: A target for neuroprotection? Lancet Neurol 8: 382-397, 2009.

42. Rossol M, Heine H, Meusch U, Quandt D, Klein C, Sweet MJ and Hauschildt S: LPS-induced cytokine production in human monocytes and macrophages. Crit Rev Immunol 31: 379-446, 2011.

43. Sarkar S, Chigurupati S, Raymick J, Mann D, Bowyer JF, Schmitt T, Beger RD, Hanig JP, Schmued LC and Paule MG: Neuroprotective effect of the chemical chaperone, trehalose in a chronic MPTP-induced Parkinson's disease mouse model. Neurotoxicology 44: 250-262, 2014.

44. Whitton PS: Inflammation as a causative factor in the aetiology of Parkinson's disease. Br J Pharmacol 150: 963-976, 2007.

45. Niranjan R: The role of inflammatory and oxidative stress mechanisms in the pathogenesis of Parkinson's disease: Focus on astrocytes. Mol Neurobiol 49: 28-38, 2014.

46. Tseng YT, Hsu YY, Shih YT and Lo YC: Paeonol attenuates microglia-mediated inflammation and oxidative stress-induced neurotoxicity in rat primary microglia and cortical neurons. Shock 37: 312-318, 2012.

47. Daubner SC, Le T and Wang S: Tyrosine hydroxylase and regulation of dopamine synthesis. Arch Biochem Biophys 508: 1-12, 2011.

48. Fukuda T, Takahashi J and Tanaka J: Tyrosine hydroxylase-immunoreactive neurons are decreased in number in the cerebral cortex of Parkinson's disease. Neuropathology 19: 10-13, 1999.

49. Episcopo FL, Tirolo C, Testa N, Caniglia S, Morale MC and Marchetti B: Reactive astrocytes are key players in nigrostriatal dopaminergic neurorepair in the MPTP mouse model of Parkinson's disease: Focus on endogenous neurorestoration. Curr Aging Sci 6: 45-55, 2013.

50. Weissmiller AM and Wu C: Current advances in using neurotrophic factors to treat neurodegenerative disorders. Transl Neurodegener 1: 14, 2012.

51. Dai L, Wang D, Meng H, Zhang K, Fu L, Wu Y and Bai Y: Association between the BDNF G196A and C270T polymorphisms and Parkinson's disease: A meta-analysis. Int J Neurosci 123: 675-683, 2013.

52. Scalzo P, Kummer A, Bretas TL, Cardoso F and Teixeira AL: Serum levels of brain-derived neurotrophic factor correlate with motor impairment in Parkinson's disease. J Neurol 257: 540-545, 2010.

53. Salehi Z and Mashayekhi F: Brain-derived neurotrophic factor concentrations in the cerebrospinal fluid of patients with Parkinson's disease. J Clin Neurosci 16: 90-93, 2009. 